International Journal of Computer Mathematics

ISSN: 0020-7160 (Print) 1029-0265 (Online) Journal homepage: https://www.tandfonline.com/loi/gcom20

\title{
Distributed fusion filtering for multi-sensor systems with correlated random transition and measurement matrices
}

Raquel Caballero-Águila, Irene García-Garrido \& Josefa Linares-Pérez

\section{Preprint version. Please cite original version:}

Raquel Caballero-Águila, Irene García-Garrido \& Josefa Linares-Pérez (2020). Distributed fusion filtering for multi-sensor systems with correlated random transition and.measurement matrices, International Journal of Computer Mathematics, 97 (1-2), 263-274.

https://doi.org/10.1080/00207160.2018.1554213

\begin{abstract}
This paper is concerned with the distributed fusion estimation problem for discrete-time linear stochastic systems with measurements coming from different sensors and correlated random parameter matrices in both the state and measurement equations. At each sampling time, the random state transition parameter matrices are assumed to be correlated at the same sampling time with the measurement random parameter matrices of each sensor. Moreover, the random parameter matrices in the observation equations are one-step auto-correlated and cross-correlated between the different sensors. The additive noises are also assumed to be correlated. Under these assumptions, the distributed fusion filter is designed as the matrix-weighted linear combination of the local least-squares linear filters obtained at every single sensor, using the linear minimum variance optimality criterion. A numerical simulation example considering a two-sensor system with randomly delayed measurements is used to illustrate the applicability of multi-sensor systems with correlated random parameter matrices and analyse the performance of the proposed filtering estimators.
\end{abstract}




\section{Introduction}

In the last decades, the estimation problem for networked stochastic systems has become a challenging research topic, due to its wide applicability in several scientific areas, mainly related to sensor and communication technologies. The most common data fusion techniques used in the state estimation problem are the centralized and distributed ones [14]. The first one provides the optimal linear filtering estimator as a combination of the measurements coming from all the sensors, so it is theoretically optimal when all the sensors work correctly. Under the distributed architecture, local filtering estimators are independently obtained at each single sensor, before being sent to a fusion center to be combined by using an optimal or suboptimal fusion criterion. This approach has considerable advantages, such as superior performance in terms of flexibility, robustness to failure and cost effectiveness. For this reason, many results on the distributed fusion estimation have been reported in recent years (see e.g. [2], [3], [11], [12] and references therein).

There are many real situations where sensor network systems are used and the unreliable network characteristics usually lead to different possible failures in the measurements caused by fading phenomena in propagation channels, degradation or attenuation of the information, inaccessibility at certain times, multiplicative noise uncertainties, among others. The distributed fusion estimation problem has been analyzed in [12] for a class of multisensor multirate systems with observation multiplicative noises. The results in [12] are extended in [15], where a distributed fusion filter is presented for multisensor multirate systems with multiplicative noises and fading measurements. The distributed $H_{\infty}$-consensus filtering problem has been addressed in [13] for a class of uncertain stochastic systems with multiple missing measurements. The fusion estimation problem has been studied in [17] when the stochastic uncertainties are caused by multiplicative noises in the state and observation matrices. In [9], the distributed fusion estimation problem has also been considered for a class of multi-sensor systems with correlated noises and fading measurements. A recursive state estimator has been designed in [8] for time-varying complex networks with missing measurements using a variance-constrained approach. The recursive finite-horizon filtering problem has been investigated in [7] for a class of nonlinear time-varying systems subject to multiplicative noises and missing measurements. Moreover, random failures or interruptions in the transmission of the measurements can occur frequently, yielding, for example, random communication packet dropouts or sensor delays. The distributed Kalman filtering problem has been addressed in [5], for a class of networked multisensor systems with missing sensor measurements, random transmission delays and packet dropouts, in [18] for stochastic non-linear systems with random delays and packet dropouts, and in [11] for uncertain networked systems considering time delays in the data transmission and cross-correlated noises.

The state estimation problem in all these types of systems can be globally addressed in a unified way by considering systems with random parameter matrices in the state and measurement equations. This kind of systems appear in a large variety of application fields, such as digital control of chemical processes, mobile robot localization, navigation systems and economics systems (see [6]) and they are currently being analyzed in many research papers. For example, systems with state-dependent multiplicative noise can be considered as a particular case of systems with random state transition parameter matrices, whereas systems with missing measurements, random delays or packet dropouts can be modeled by systems with measurement random parameter matrices. The study of the centralized and distributed fusion estimation problems in 
systems with random parameter matrices has been addressed under different correlation assumptions on the random parameter matrices and the noises involved in the system model. The centralized fusion estimation problem has been studied in [10] and the distributed fusion estimation problem has been analyzed in [2] for discretetime linear stochastic systems with multi-sensor measurements including correlated and cross-correlated random parameter matrices in the measurement equations and noises, and the results obtained have been applied to systems with correlated missing and randomly delayed measurements. Also, the fusion estimation problem from measurements subject to random delays and packet dropouts during the transmission has been studied in [4]. More recently, in [16] the centralized estimation problem has been investigated for a class of non-linear discrete-time stochastic systems with random parameter matrices which are correlated with each other at the same time, fading measurements and correlated noises.

Motivated by the above discussion, this paper is concerned with the distributed fusion estimation problem for discrete-time linear stochastic systems with measurements coming from different sensors, correlated random parameter matrices in both, the state and measurement equations, and correlated noises. The main contributions of this paper are summarized as follows: (a) The system model is quite comprehensive, since it covers general situations involving correlated multiplicative noises and other phenomena, such as correlated missing measurements or delayed measurements, among others. (b) The proposed results are more general than those in [2], where the distributed fusion filters are obtained for networked systems with independent random state transition matrices, whereas correlation at the same sampling time with the measurement random parameter matrices of each sensor is considered in the current paper. (c) The proposed recursive algorithm for the local least-squares linear filters is derived by using the innovation approach and the distributed fusion filter is designed as the matrix weighted linear combination of the local ones which minimizes the mean squared filtering estimation error. This methodology is very simple computationally and suitable for online applications.

The rest of the paper is organized as follows. In Section 2 the system model is established and the distributed estimation problem is formulated. In Section 3, the local least-squares linear filtering algorithm is given, the cross-covariance matrices between any two local filters are presented, and the proposed distributed fusion filter is generated as a matrix-weighted linear combination of the local filters using the mean squared error optimality criterion. The numerical simulation example in Section 4 shows how the current model is applicable to multi-sensor systems with correlated random delays in transmission and analyzes the effectiveness of the proposed distributed fusion filter. The paper ends with some concluding remarks in Section 5.

\section{Problem formulation}

Consider a class of discrete-time linear stochastic systems with $n_{x}$-dimensional state process, $\left\{x_{k}\right\}_{k \geq 0}$, whose evolution is described by random transition parameter matrices, $\left\{F_{k}\right\}_{k \geq 0}$, and an additive process noise, $\left\{w_{k}\right\}_{k \geq 0}$. Specifically, the state equation is given by

$$
x_{k+1}=F_{k} x_{k}+w_{k}, \quad k \geq 0 .
$$

Assuming that there are $m$ sensors from which the state process is observed, the 
$n_{y}$-dimensional measurements provided by the $i$-th sensor, $\left\{y_{k}^{(i)}\right\}_{k \geq 1}$, are described as follows:

$$
y_{k}^{(i)}=H_{k}^{(i)} x_{k}+B_{k}^{(i)} v_{k}^{(i)}, \quad k \geq 1, \quad i=1, \ldots, m
$$

where $\left\{H_{k}^{(i)}\right\}_{k \geq 1}$ and $\left\{B_{k}^{(i)}\right\}_{k \geq 1}$ are sequences of random parameter matrices, and $\left\{v_{k}^{(i)}\right\}_{k \geq 1}$ is the $n_{v}$-dimensional process noise of the $i$-th sensor.

Our aim is to solve the distributed fusion estimation problem of the state $x_{k}$ based on the measurements coming from all the sensors, $y_{1}^{(i)}, y_{2}^{(i)}, \ldots, y_{k}^{(i)}, i=1, \ldots, m$, under the following hypotheses about the initial state, the random parameter matrices and the noise processes involved in the previous system (1)-(2):

Hypothesis 1 . The initial state $x_{0}$ is a zero-mean random vector with $\operatorname{Cov}\left[x_{0}\right]=\Sigma_{0}$. It is also assumed to be independent of the random parameter matrices and noise processes.

Hypothesis 2. The mean functions of $\left\{F_{k}\right\}_{k \geq 0},\left\{H_{k}^{(i)}\right\}_{k \geq 1}$ and $\left\{B_{k}^{(i)}\right\}_{k \geq 1}, i=$ $1, \ldots, m$, are known. We will denote $\bar{F}_{k} \equiv E\left[F_{k}\right], k \geq 0, \bar{H}_{k}^{(i)} \equiv E\left[H_{k}^{(i)}\right]$ and $\bar{B}_{k}^{(i)} \equiv E\left[B_{k}^{(i)}\right], k \geq 1$. For $i, j=1, \ldots, m$, the following expectations are also assumed to be known:

$$
\begin{aligned}
& E\left[f_{p q}(k) f_{p^{\prime} q^{\prime}}(s)\right]=E\left[f_{p q}(k) f_{p^{\prime} q^{\prime}}(k)\right] \delta_{k, s}, \\
& E\left[h_{p q}^{(i)}(k) h_{p^{\prime} q^{\prime}}^{(j)}(s)\right]=E\left[h_{p q}^{(i)}(k) h_{p^{\prime} q^{\prime}}^{(j)}(k)\right] \delta_{k, s}+E\left[h_{p q}^{(i)}(k) h_{p^{\prime} q^{\prime}}^{(j)}(k-1)\right] \delta_{k-1, s}, \quad s \leq k, \\
& E\left[b_{p q}^{(i)}(k) b_{p^{\prime} q^{\prime}}^{(j)}(s)\right]=E\left[b_{p q}^{(i)}(k) b_{p^{\prime} q^{\prime}}^{(j)}(k)\right] \delta_{k, s}+E\left[b_{p q}^{(i)}(k) b_{p^{\prime} q^{\prime}}^{(j)}(k-1)\right] \delta_{k-1, s}, \quad s \leq k, \\
& E\left[f_{p q}(k) h_{p^{\prime} q^{\prime}}^{(i)}(s)\right]=E\left[f_{p q}(k) h_{p^{\prime} q^{\prime}}^{(i)}(k)\right] \delta_{k, s}, \\
& E\left[f_{p q}(k) b_{p^{\prime} q^{\prime}}^{(i)}(s)\right]=E\left[f_{p q}(k) b_{p^{\prime} q^{\prime}}^{(i)}(k)\right] \delta_{k, s}, \\
& E\left[h_{p q}^{(i)}(k) b_{p^{\prime} q^{\prime}}^{(j)}(s)\right]=E\left[h_{p q}^{(i)}(k) b_{p^{\prime} q^{\prime}}^{(j)}(k)\right] \delta_{k, s}+E\left[h_{p q}^{(i)}(k) b_{p^{\prime} q^{\prime}}^{(j)}(k-1)\right] \delta_{k-1, s} \\
& \quad+E\left[h_{p q}^{(i)}(k) b_{p^{\prime} q^{\prime}}^{(j)}(k+1)\right] \delta_{k+1, s}, \\
& E\left[h_{p q}^{(i)}(k) f_{q r}(s) h_{p^{\prime} q^{\prime}}^{(j)}(s)\right]=E\left[h_{p q}^{(i)}(k) f_{q r}(k-1) h_{p^{\prime} q^{\prime}}^{(j)}(k-1)\right] \delta_{k-1, s}, \\
& E\left[h_{p q}^{(i)}(k) f_{q r}(s) b_{p^{\prime} q^{\prime}}^{(j)}(s)\right]=E\left[h_{p q}^{(i)}(k) f_{q r}(k-1) b_{p^{\prime} q^{\prime}}^{(j)}(k-1)\right] \delta_{k-1, s},
\end{aligned}
$$

where $\delta_{k, s}$ is the Kronecker delta function and $f_{p q}(r), h_{p q}^{(i)}(r)$ and $b_{p q}^{(i)}(r)$ denote the $(p, q)$-th entries of $F_{r}, H_{r}^{(i)}$ and $B_{r}^{(i)}$, respectively.

Usually, in practical situations, the probability distributions of these random variables describing the entries of the random parameter matrices, $f_{p q}(r), h_{p q}^{(i)}(r)$ and $b_{p q}^{(i)}(r)$, are known and such distributions, together with the relation between these entries, provide the necessary information to calculate these expectations.

From this hypothesis, the random parameter matrices are correlated with each other. Specifically, the random state transition matrices, $\left\{F_{k}\right\}_{k \geq 0}$, and the measurement matrices, $\left\{H_{k}^{(i)}\right\}_{k \geq 1}$ and $\left\{B_{k}^{(i)}\right\}_{k \geq 1}$ of each sensor, are correlated at the same sampling time. Furthermore, the measurement matrices are assumed to be one-step correlated and cross-correlated between the different sensors. This correlation hypothesis covers situations involving correlated multiplicative noises, such as interferences or intermit- 
tent sensor failures. Also, transformed versions from networked systems with random delays and packet dropouts include correlated random parameter matrices. Therefore, by virtue of Hypothesis 2 the estimation algorithm designed in this paper can be applied to all these situations involving random correlated uncertainties.

Hypothesis 3. $\left\{w_{k}\right\}_{k \geq 0}$ and $\left\{v_{k}^{(i)}\right\}_{k \geq 1}, i=1, \ldots, m$, are zero-mean sequences with the following known covariances and cross-covariances:

$$
\begin{aligned}
& E\left[w_{k} w_{s}^{T}\right]=Q_{k} \delta_{k, s}+Q_{k, k-1} \delta_{k-1, s}, \quad s \leq k, \\
& E\left[v_{k}^{(i)} v_{s}^{(j) T}\right]=R_{k}^{(i j)} \delta_{k, s}+R_{k, k-1}^{(i j)} \delta_{k-1, s}, \quad s \leq k, \quad i, j=1, \ldots, m, \\
& E\left[w_{k} v_{s}^{(i) T}\right]=S_{k}^{(i)} \delta_{k, s}+S_{k, k+1}^{(i)} \delta_{k+1, s}+S_{k, k+2}^{(i)} \delta_{k+2, s}, \quad k \geq 0, \quad s \geq 1, \quad i=1, \ldots, m .
\end{aligned}
$$

This hypothesis indicates that the process and measurement noises are one-step autocorrelated, and two-step forward cross-correlated. The measurement noises are also one-step cross-correlated between the different sensors.

Hypothesis 4. $\left(\left\{F_{k}\right\}_{k \geq 0},\left\{H_{k}^{(i)}\right\}_{k \geq 1},\left\{B_{k}^{(i)}\right\}_{k \geq 1}, i=1, \ldots, m\right)$ is independent of $\left(\left\{w_{k}\right\}_{k \geq 0},\left\{v_{k}^{(i)}\right\}_{k \geq 1}, i=1, \ldots, m\right)$.

\section{Design of distributed fusion filters}

In this section, we design the distributed fusion filter of the state $x_{k}$ based on the available measurements $y_{1}^{(i)}, \ldots, y_{k}^{(i)}, i=1, \ldots, m$. First, local least-squares linear filters are obtained at each sensor, using its own measurement information by means of a recursive algorithm. Then, the cross-covariance matrices between any two local filters are presented. To conclude, the distributed fusion filter is obtained by applying the optimal information fusion criterion weighted by matrices in the linear minimum variance sense.

\subsection{Local least-squares linear filters}

The following theorem provides the recursive algorithm for the local least-squares linear filter and its error covariance matrix at each sensor $i=1, \ldots, m$.

Theorem 3.1. For system (1)-(2), under hypotheses 1-4, the local least-squares linear filter for the $i$-th sensor, $\widehat{x}_{k / k}^{(i)}, i=1, \ldots, m$, is obtained as

$$
\widehat{x}_{k / k}^{(i)}=\widehat{x}_{k / k-1}^{(i)}+\mathcal{X}_{k}^{(i)} \Pi_{k}^{(i)-1} \mu_{k}^{(i)}, \quad k \geq 1 ; \quad \widehat{x}_{0 / 0}^{(i)}=0 .
$$

The one-step predictor, $\widehat{x}_{k / k-1}^{(i)}$, is calculated by

$$
\widehat{x}_{k / k-1}^{(i)}=\bar{F}_{k-1} \widehat{x}_{k-1 / k-1}^{(i)}+\Phi_{k-1}^{(i)} \Pi_{k-1}^{(i)-1} \mu_{k-1}^{(i)}, \quad k \geq 2 ; \quad \widehat{x}_{1 / 0}^{(i)}=0,
$$


where, denoting $\widetilde{F}_{k} \equiv F_{k}-\bar{F}_{k}$, the matrix $\Phi_{k}^{(i)} \equiv E\left[\left(\widetilde{F}_{k} x_{k}+w_{k}\right) \mu_{k}^{(i) T}\right]$ is given by

$$
\Phi_{k}^{(i)}=E\left[\widetilde{F}_{k} \mathcal{D}_{k} H_{k}^{(i) T}\right]+E\left[\widetilde{F}_{k} \mathcal{E}_{k}^{(i)} B_{k}^{(i) T}\right]+Q_{k, k-1} \bar{H}_{k}^{(i) T}+S_{k}^{(i)} \bar{B}_{k}^{(i) T}, \quad k \geq 1 .
$$

The innovation, $\mu_{k}^{(i)}$, is given by

$$
\mu_{k}^{(i)}=y_{k}^{(i)}-\bar{H}_{k}^{(i)} \widehat{x}_{k / k-1}^{(i)}-\Psi_{k, k-1}^{(i)} \Pi_{k-1}^{(i)-1} \mu_{k-1}^{(i)}, \quad k \geq 2 ; \quad \mu_{1}^{(i)}=y_{1}^{(i)},
$$

where, denoting $\widetilde{H}_{k}^{(i)} \equiv H_{k}^{(i)}-\bar{H}_{k}^{(i)}$, the matrix $\Psi_{k, k-1}^{(i)} \equiv E\left[\left(\widetilde{H}_{k}^{(i)} x_{k}+B_{k}^{(i)} v_{k}^{(i)}\right) \mu_{k-1}^{(i) T}\right]$ satisfies

$$
\begin{aligned}
\Psi_{k, k-1}^{(i)}= & E\left[\widetilde{H}_{k}^{(i)} F_{k-1} \mathcal{D}_{k-1} H_{k-1}^{(i) T}\right]+E\left[\widetilde{H}_{k}^{(i)} Q_{k-1, k-2} H_{k-1}^{(i) T}\right] \\
& +E\left[\widetilde{H}_{k}^{(i)} F_{k-1} \mathcal{E}_{k-1}^{(i)} B_{k-1}^{(i) T}\right]+E\left[\widetilde{H}_{k}^{(i)} S_{k-1}^{(i)} B_{k-1}^{(i) T}\right] \\
& +E\left[B_{k}^{(i)} S_{k-2, k}^{(i) T} H_{k-1}^{(i) T}\right]+E\left[B_{k}^{(i)} R_{k, k-1}^{(i)} B_{k-1}^{(i) T}\right], \quad k \geq 2
\end{aligned}
$$

The matrix $\mathcal{X}_{k}^{(i)} \equiv E\left[x_{k} \mu_{k}^{(i) T}\right]$ is calculated by

$$
\begin{aligned}
\mathcal{X}_{k}^{(i)} & =\Sigma_{k / k-1}^{(i)} \bar{H}_{k}^{(i) T}+\mathcal{E}_{k}^{(i)} \bar{B}_{k}^{(i) T}-\mathcal{X}_{k, k-1}^{(i)} \Pi_{k-1}^{(i)-1} \Psi_{k, k-1}^{(i) T}, \quad k \geq 2 \\
\mathcal{X}_{1}^{(i)} & =\Sigma_{1 / 0}^{(i)} \bar{H}_{1}^{(i) T}+\mathcal{E}_{1}^{(i)} \bar{B}_{1}^{(i) T},
\end{aligned}
$$

where $\mathcal{X}_{k, k-1}^{(i)} \equiv E\left[x_{k} \mu_{k-1}^{(i) T}\right]$ is given by

$$
\mathcal{X}_{k, k-1}^{(i)}=\bar{F}_{k-1} \mathcal{X}_{k-1}^{(i)}+\Phi_{k-1}^{(i)}, \quad k \geq 2
$$

The innovation covariance matrix, $\Pi_{k}^{(i)} \equiv E\left[\mu_{k}^{(i)} \mu_{k}^{(i) T}\right]$, satisfies

$$
\begin{aligned}
\Pi_{k}^{(i)}= & E\left[\widetilde{H}_{k}^{(i)} \mathcal{D}_{k} H_{k}^{(i) T}\right]+E\left[\widetilde{H}_{k}^{(i)} \mathcal{E}_{k}^{(i)} B_{k}^{(i) T}\right]+E\left[B_{k}^{(i)} \mathcal{E}_{k}^{(i) T} \widetilde{H}_{k}^{(i) T}\right]+E\left[B_{k}^{(i)} R_{k}^{(i i)} B_{k}^{(i) T}\right] \\
& +\bar{H}_{k}^{(i)} \mathcal{X}_{k}^{(i)}+\mathcal{X}_{k}^{(i) T} \bar{H}_{k}^{(i) T}-\bar{H}_{k}^{(i)} \Sigma_{k / k-1}^{(i)} \bar{H}_{k}^{(i) T}-\Psi_{k, k-1}^{(i)} \Pi_{k-1}^{(i)-1} \Psi_{k, k-1}^{(i) T}, \quad k \geq 2 ; \\
\Pi_{1}^{(i)}= & E\left[\widetilde{H}_{1}^{(i)} \mathcal{D}_{1} H_{1}^{(i) T}\right]+E\left[H_{1}^{(i)} \mathcal{E}_{1}^{(i)} B_{1}^{(i) T}\right]+E\left[B_{1}^{(i)} \mathcal{E}_{1}^{(i) T} H_{1}^{(i) T}\right] \\
& +E\left[B_{1}^{(i)} R_{1}^{(i i)} B_{1}^{(i) T}\right]+\bar{H}_{1}^{(i)} \Sigma_{1 / 0}^{(i)} \bar{H}_{1}^{(i) T} .
\end{aligned}
$$

The filtering error covariance matrix, $\Sigma_{k / k}^{(i)} \equiv E\left[\left(x_{k}-\widehat{x}_{k / k}^{(i)}\right)\left(x_{k}-\widehat{x}_{k / k}^{(i)}\right)^{T}\right]$, is computed by

$$
\Sigma_{k / k}^{(i)}=\Sigma_{k / k-1}^{(i)}-\mathcal{X}_{k}^{(i)} \Pi_{k}^{(i)-1} \mathcal{X}_{k}^{(i) T}, \quad k \geq 1 ; \quad \Sigma_{0 / 0}^{(i)}=\Sigma_{0}
$$

where the prediction error covariance matrix, $\Sigma_{k / k-1}^{(i)} \equiv E\left[\left(x_{k}-\widehat{x}_{k / k-1}^{(i)}\right)\left(x_{k}-\widehat{x}_{k / k-1}^{(i)}\right)^{T}\right]$, 
is given by

$$
\begin{aligned}
\Sigma_{k / k-1}^{(i)}= & \mathcal{D}_{k}+\bar{F}_{k-1}\left(\Sigma_{k-1 / k-1}^{(i)}-\mathcal{D}_{k-1}\right) \bar{F}_{k-1}^{T}+\Phi_{k-1}^{(i)} \Pi_{k-1}^{(i)-1} \Phi_{k-1}^{(i) T} \\
& -\mathcal{X}_{k, k-1}^{(i)} \Pi_{k-1}^{(i)-1} \Phi_{k-1}^{(i) T}-\Phi_{k-1}^{(i)} \Pi_{k-1}^{(i)-1} \mathcal{X}_{k, k-1}^{(i) T}, \quad k \geq 2 ; \\
\Sigma_{1 / 0}^{(i)}= & \mathcal{D}_{1} .
\end{aligned}
$$

Finally, the matrix $\mathcal{D}_{k+1} \equiv E\left[x_{k+1} x_{k+1}^{T}\right]$ is calculated by

$$
\begin{aligned}
& \mathcal{D}_{k+1}=E\left[F_{k} \mathcal{D}_{k} F_{k}^{T}\right]+Q_{k}+\bar{F}_{k} Q_{k-1, k}+Q_{k, k-1} \bar{F}_{k}^{T}, \quad k \geq 1 ; \\
& \mathcal{D}_{1}=E\left[F_{0} \mathcal{D}_{0} F_{0}^{T}\right]+Q_{0} ; \quad \mathcal{D}_{0}=\Sigma_{0},
\end{aligned}
$$

and $\mathcal{E}_{k}^{(i)} \equiv E\left[x_{k} v_{k}^{(i) T}\right]$ is obtained by

$$
\mathcal{E}_{k}^{(i)}=\bar{F}_{k-1} S_{k-2, k}^{(i)}+S_{k-1, k}^{(i)}, k \geq 2 ; \quad \mathcal{E}_{1}^{(i)}=S_{0,1}^{(i)}
$$

Proof. The proof, based on the innovation approach, is analogous to that in Section 3 of [10] except for the calculation of the matrices $\Phi_{k}^{(i)}$ and $\Psi_{k, k-1}^{(i)}$ because of the correlation between $\left\{F_{k}\right\}_{k \geq 0},\left\{H_{k}\right\}_{k \geq 1}$ and $\left\{B_{k}\right\}_{k \geq 1}$. Thus, only expressions (3) and (5) for these matrices are proved:

(I) Derivation of expression (3) for $\Phi_{k}^{(i)} \equiv E\left[\left(\widetilde{F}_{k} x_{k}+w_{k}\right) \mu_{k}^{(i) T}\right]$.

Since $\widetilde{F}_{k} x_{k}+w_{k}$ is independent of $y_{1}^{(i)}, \ldots, y_{k-1}^{(i)}$, we have $\Phi_{k}^{(i)}=E\left[\left(\widetilde{F}_{k} x_{k}+\right.\right.$ $\left.w_{k}\right) y_{k}^{(i) T}$. From (2) for $y_{k}^{(i)}$, we obtain that

$$
\Phi_{k}^{(i)}=E\left[\widetilde{F}_{k} x_{k} x_{k}^{T} H_{k}^{(i) T}\right]+E\left[\widetilde{F}_{k} x_{k} v_{k}^{(i) T} B_{k}^{(i) T}\right]+E\left[w_{k} x_{k}^{T} H_{k}^{(i) T}\right]+E\left[w_{k} v_{k}^{(i) T} B_{k}^{(i) T}\right]
$$

Taking into account the conditional expectation properties, we have $E\left[\widetilde{F}_{k} x_{k} x_{k}^{T} H_{k}^{(i) T}\right]=E\left[\widetilde{F}_{k} \mathcal{D}_{k} H_{k}^{(i) T}\right]$ and $E\left[\widetilde{F}_{k} x_{k} v_{k}^{(i) T} B_{k}^{(i) T}\right]=E\left[\widetilde{F}_{k} \mathcal{E}_{k}^{(i)} B_{k}^{(i) T}\right]$. The noise correlation hypothesis together with (1) yield $E\left[w_{k} x_{k}^{T} H_{k}^{(i) T}\right]=Q_{k, k-1} \bar{H}_{k}^{(i) T}$ and $E\left[w_{k} v_{k}^{(i) T} B_{k}^{(i) T}\right]=S_{k}^{(i)} \bar{B}_{k}^{(i) T}$. Therefore, from the above expectations, expression (3) for $\Phi_{k}^{(i)}$ is clear.

(II) Derivation of expression (5) for $\Psi_{k, k-1}^{(i)} \equiv E\left[\left(\widetilde{H}_{k}^{(i)} x_{k}+B_{k}^{(i)} v_{k}^{(i)}\right) \mu_{k-1}^{(i) T}\right]$.

Following a similar reasoning to the previous one and using (1) for $x_{k}$ in two steps, we obtain that

$$
\begin{aligned}
\Psi_{k, k-1}^{(i)}= & E\left[\widetilde{H}_{k} x_{k} x_{k-1}^{T} H_{k-1}^{(i) T}\right]+E\left[\widetilde{H}_{k} x_{k} v_{k-1}^{(i) T} B_{k-1}^{(i) T}\right] \\
& +E\left[B_{k}^{(i)} v_{k}^{(i)} x_{k-1}^{T} H_{k-1}^{(i) T}\right]+E\left[B_{k}^{(i)} v_{k}^{(i)} v_{k-1}^{(i) T} B_{k-1}^{(i) T}\right] \\
= & E\left[\widetilde{H}_{k} F_{k-1} \mathcal{D}_{k-1} H_{k-1}^{(i) T}\right]+E\left[\widetilde{H}_{k} w_{k-1} x_{k-1}^{T} H_{k-1}^{(i) T}\right] \\
& +E\left[\widetilde{H}_{k} F_{k-1} \mathcal{E}_{k-1}^{(i)} B_{k-1}^{(i) T}\right]+E\left[\widetilde{H}_{k} S_{k-1}^{(i)} B_{k-1}^{(i) T}\right] \\
& +E\left[B_{k}^{(i)} v_{k}^{(i)} x_{k-1}^{T} H_{k-1}^{(i) T}\right]+E\left[B_{k}^{(i)} R_{k, k-1}^{(i)} B_{k-1}^{(i) T}\right] .
\end{aligned}
$$


Then, considering again (1) and hypothesis 3, it is deduced that

$$
\begin{aligned}
& E\left[\widetilde{H}_{k} w_{k-1} x_{k-1}^{T} H_{k-1}^{(i) T}\right]=E\left[\widetilde{H}_{k} Q_{k-1, k-2} H_{k-1}^{(i) T}\right], \\
& E\left[B_{k}^{(i)} v_{k}^{(i)} x_{k-1}^{T} H_{k-1}^{(i) T}\right]=E\left[B_{k}^{(i)} S_{k-2, k}^{(i) T} H_{k-1}^{(i) T}\right],
\end{aligned}
$$

so, expression (5) for $\Psi_{k, k-1}^{(i)}$ is also immediately clear, and the proof is completed.

\subsection{Cross-covariance matrices between any two local filters}

To apply the optimal fusion criterion weighted by matrices in the linear minimum variance sense, the cross-covariance matrices between local estimators of any two subsystems must be calculated.

Theorem 3.2. For $i, j=1, \ldots, m$, the cross-covariance matrices, $K_{k / k}^{(i j)} \equiv$ $E\left[\widehat{x}_{k / k}^{(i)} \widehat{x}_{k / k}^{(j) T}\right]$ between any two local filters are calculated as

$$
\begin{aligned}
K_{k / k}^{(i j)}= & K_{k / k-1}^{(i j)}+L_{k}^{(i j)} \Pi_{k}^{(j)-1} \mathcal{X}_{k}^{(j) T}+\mathcal{X}_{k}^{(i)} \Pi_{k}^{(i)-1} L_{k}^{(j i) T} \\
& +\mathcal{X}_{k}^{(i)} \Pi_{k}^{(i)-1} \Pi_{k}^{(i j)} \Pi_{k}^{(j)-1} \mathcal{X}_{k}^{(j) T}, \quad k \geq 1, i \neq j, \quad K_{k / k}^{(i i)}=\mathcal{D}_{k}-\Sigma_{k / k}^{(i)}, \quad k \geq 1,
\end{aligned}
$$

where

(a) For $i, j=1, \ldots, m, K_{k / k-1}^{(i j)} \equiv E\left[\widehat{x}_{k / k-1}^{(i)} \widehat{x}_{k / k-1}^{(j) T}\right]$ is recursively calculated by

$$
\begin{aligned}
K_{k / k-1}^{(i j)}= & \bar{F}_{k-1} K_{k-1 / k-2}^{(i j)} \bar{F}_{k-1}^{T}+\bar{F}_{k-1} L_{k-1}^{(i j)} \Pi_{k-1}^{(j)-1} \mathcal{X}_{k, k-1}^{(j) T} \\
& +\mathcal{X}_{k, k-1}^{(i)} \Pi_{k-1}^{(i)-1} L_{k-1}^{(j i) T} \bar{F}_{k-1}^{T} \\
& +\mathcal{X}_{k, k-1}^{(i)} \Pi_{k-1}^{(i)-1} \Pi_{k-1}^{(i j)} \Pi_{k-1}^{(j)-1} \mathcal{X}_{k, k-1}^{(j) T}, \quad k \geq 2, \quad i \neq j ; \\
K_{1 / 0}^{(i j)}=0 & ; \\
K_{k / k-1}^{(i i)}= & \mathcal{D}_{k}-\Sigma_{k / k-1}^{(i)}, \quad k \geq 1 .
\end{aligned}
$$

(b) For $i, j=1, \ldots, m$, with $i \neq j, L_{k}^{(i j)} \equiv E\left[\widehat{x}_{k / k-1}^{(i)} \mu_{k}^{(j) T}\right]$ is given by

$$
\begin{aligned}
L_{k}^{(i j)}= & \left(\bar{F}_{k-1} K_{k-1 / k-2}^{(i i)} \bar{F}_{k-1}^{T}-K_{k / k-1}^{(i j)}\right) \bar{H}_{k}^{(j) T} \\
& +\mathcal{X}_{k, k-1}^{(i)} \Pi_{k-1}^{(i)-1}\left(\Delta_{k, k-1}^{(j i) T}+\mathcal{V}_{k, k-1}^{(j i) T}\right)-L_{k, k-1}^{(i j)} \Pi_{k-1}^{(j)-1} \Psi_{k, k-1}^{(j) T}, \quad k \geq 2 ; \\
L_{1}^{(i j)}= & 0,
\end{aligned}
$$

where 
- $\Delta_{k, k-1}^{(i j)} \equiv E\left[H_{k}^{(i)} x_{k} \mu_{k-1}^{(j) T}\right]$ is obtained by

$$
\begin{aligned}
& \Delta_{k, k-1}^{(i j)}= E\left[H_{k}^{(i)} F_{k-1} \mathcal{D}_{k-1} H_{k-1}^{(j) T}\right]+E\left[H_{k}^{(i)} Q_{k-1, k-2} H_{k-1}^{(j) T}\right] \\
&+E\left[H_{k}^{(i)} F_{k-1} \mathcal{E}_{k-1}^{(j)} B_{k-1}^{(j) T}\right]+E\left[H_{k}^{(i)} S_{k-1}^{(j)} B_{k-1}^{(j) T}\right] \\
&-\bar{H}_{k}^{(i)} \bar{F}_{k-1}\left(K_{k-1 / k-2}^{(j j)} \bar{H}_{k-1}^{(j) T}+\mathcal{X}_{k-1, k-2}^{(j)} \Pi_{k-2}^{(j)-1} \Psi_{k-1, k-2}^{(j) T}\right), k \geq 3 ; \\
& \Delta_{2,1}^{(i j)}=E\left[H_{2}^{(i)} F_{1} \mathcal{D}_{1} H_{1}^{(j) T}\right]+E\left[H_{2}^{(i)} Q_{1,0} H_{1}^{(j) T}\right] \\
&+E\left[H_{2}^{(i)} F_{1} \mathcal{E}_{1}^{(j)} B_{1}^{(j) T}\right]+E\left[H_{2}^{(i)} S_{1}^{(j)} B_{1}^{(j) T}\right],
\end{aligned}
$$

- $\mathcal{V}_{k, k-1}^{(i j)} \equiv E\left[B_{k}^{(i)} v_{k}^{(i)} y_{k-1}^{(j) T}\right]$ is given by

$$
\mathcal{V}_{k, k-1}^{(i j)}=E\left[B_{k}^{(i)} S_{k-2, k}^{(i) T} H_{k-1}^{(j) T}\right]+E\left[B_{k}^{(i)} R_{k, k-1}^{(i j)} B_{k-1}^{(j) T}\right], \quad k \geq 2,
$$

- $L_{k, k-1}^{(i j)} \equiv E\left[\widehat{x}_{k / k-1}^{(i)} \mu_{k-1}^{(j) T}\right]$ is calculated by

$$
L_{k, k-1}^{(i j)}=\bar{F}_{k-1} L_{k-1}^{(i j)}+\mathcal{X}_{k, k-1}^{(i)} \Pi_{k-1}^{(i)-1} \Pi_{k-1}^{(i j)}, \quad k \geq 2 .
$$

(c) For $i, j=1, \ldots, m$, with $i \neq j, \Pi_{k}^{(i j)} \equiv E\left[\mu_{k}^{(i)} \mu_{k}^{(j) T}\right]$ is expressed by

$$
\begin{aligned}
\Pi_{k}^{(i j)}= & \Delta_{k}^{(i j)}+E\left[B_{k}^{(i)} \mathcal{E}_{k}^{(i) T} H_{k}^{(j) T}\right]+E\left[B_{k}^{(i)} R_{k}^{(i j)} B_{k}^{(j) T}\right]-\bar{H}_{k}^{(i)} L_{k}^{(i j)} \\
& -\mathcal{V}_{k, k-1}^{(i j)} \Pi_{k-1}^{(j)-1}\left(\bar{H}_{k}^{(j)} \mathcal{X}_{k, k-1}^{(j)}+\Psi_{k, k-1}^{(j)}\right)^{T}-\Psi_{k, k-1}^{(i)} \Pi_{k-1}^{(i)-1} \Pi_{k-1, k}^{(i j)}, \quad k \geq 2 ; \\
\Pi_{1}^{(i j)}= & \Delta_{1}^{(i j)}+E\left[B_{1}^{(i)} \mathcal{E}_{1}^{(i) T} H_{1}^{(j) T}\right]+E\left[B_{1}^{(i)} R_{1}^{(i j)} B_{1}^{(j) T}\right],
\end{aligned}
$$

where

- $\Delta_{k}^{(i j)} \equiv E\left[H_{k}^{(i)} x_{k} \mu_{k}^{(j) T}\right]$ satisfies

$$
\begin{aligned}
\Delta_{k}^{(i j)}= & E\left[H_{k}^{(i)} \mathcal{D}_{k} H_{k}^{(j) T}\right]+E\left[H_{k}^{(i)} \mathcal{E}_{k}^{(j)} B_{k}^{(j) T}\right]-\bar{H}_{k}^{(i)} \bar{F}_{k-1} K_{k-1 / k-2}^{(j j)} \bar{F}_{k-1}^{T} \bar{H}_{k}^{(j) T} \\
& -\Delta_{k, k-1}^{(i j)} \Pi_{k-1}^{(j)-1}\left(\bar{H}_{k}^{(j)} \mathcal{X}_{k, k-1}^{(j)}+\Psi_{k, k-1}^{(j)}\right)^{T}, \quad k \geq 2 \\
\Delta_{1}^{(i j)}= & E\left[H_{1}^{(i)} \mathcal{D}_{1} H_{1}^{(j) T}\right]+E\left[H_{1}^{(i)} \mathcal{E}_{1}^{(j)} B_{1}^{(j) T}\right]
\end{aligned}
$$

- $\Pi_{k-1, k}^{(i j)} \equiv E\left[\mu_{k-1}^{(i)} \mu_{k}^{(j) T}\right]$ is calculated by

$$
\Pi_{k-1, k}^{(i j)}=\Delta_{k, k-1}^{(j i) T}+\mathcal{V}_{k, k-1}^{(j i) T}-L_{k, k-1}^{(j i) T} \bar{H}_{k}^{(j) T}-\Pi_{k-1}^{(i j)} \Pi_{k-1}^{(j)-1} \Psi_{k, k-1}^{(j) T}, \quad k \geq 2 .
$$

Finally, for $i=1, \ldots, m, \Pi_{k}^{(i)}, \mathcal{X}_{k}^{(i)}, \Sigma_{k / k}^{(i)}$ and $\mathcal{D}_{k}$ are specified in Theorem 3.1.

Proof. The proof is similar to that in Section 3.2 of [2], except for the derivation of the expectation $\Delta_{k, k-1}^{(i j)} \equiv E\left[H_{k}^{(i)} x_{k} \mu_{k-1}^{(j) T}\right]$, which is affected by the correlation of the transition matrices $\left\{F_{k}\right\}_{k \geq 0}$. For this reason, only expression (6) for $\Delta_{k, k-1}^{(i j)}$ is proved 
here. Firstly, substituting (4) for $\mu_{k-1}^{(j)}$, we deduce that $\Delta_{k, k-1}^{(i j)}$ can be written as:

$$
\Delta_{k, k-1}^{(i j)}=E\left[H_{k}^{(i)} x_{k} y_{k-1}^{(j) T}\right]-E\left[H_{k}^{(i)} x_{k} \widehat{x}_{k-1 / k-2}^{(j) T}\right] \bar{H}_{k-1}^{(j) T}-E\left[H_{k}^{(i)} x_{k} \mu_{k-2}^{(j) T}\right] \Pi_{k-2}^{(j)-1} \Psi_{k-1, k-2}^{(j) T} .
$$

Secondly, using Equation (1) for $x_{k}$ and considering the conditional expectation properties, we have

$$
\begin{aligned}
\Delta_{k, k-1}^{(i j)}= & E\left[H_{k}^{(i)} F_{k-1} \mathcal{D}_{k-1} H_{k-1}^{(j) T}\right]+E\left[H_{k}^{(i)} w_{k-1} x_{k-1}^{T} H_{k-1}^{(j) T}\right] \\
& +E\left[H_{k}^{(i)} F_{k-1} \mathcal{E}_{k-1}^{(j)} B_{k-1}^{(j) T}\right]+E\left[H_{k}^{(i)} S_{k-1}^{(j)} B_{k-1}^{(j) T}\right] \\
& -E\left[H_{k}^{(i)} F_{k-1} x_{k-1} \widehat{x}_{k-1 / k-2}^{(j) T}\right] \bar{H}_{k-1}^{(j) T}-\bar{H}_{k}^{(i)} E\left[x_{k} \mu_{k-2}^{(j) T}\right] \Pi_{k-2}^{(j)-1} \Psi_{k-1, k-2}^{(j) T}
\end{aligned}
$$

Next, to obtain $E\left[H_{k}^{(i)} w_{k-1} x_{k-1}^{T} H_{k-1}^{(j) T}\right]$ and $E\left[x_{k} \mu_{k-2}^{(j) T}\right]$, we use again Equation (1) for $x_{k-1}$ and $x_{k}$, and it is easy to see that

$$
\begin{aligned}
& E\left[H_{k}^{(i)} w_{k-1} x_{k-1}^{T} H_{k-1}^{(j) T}\right]=E\left[H_{k}^{(i)} Q_{k-1, k-2} H_{k-1}^{(j) T}\right], \\
& E\left[x_{k} \mu_{k-2}^{(j) T}\right]=\bar{F}_{k-1} \mathcal{X}_{k-1, k-2}^{(i)}
\end{aligned}
$$

Finally, from the Orthogonal Projection Lemma, it is derived that $E\left[H_{k}^{(i)} F_{k-1} x_{k-1} \widehat{x}_{k-1 / k-2}^{(j) T}\right]=\bar{H}_{k}^{(i)} \bar{F}_{k-1} K_{k-1 / k-2}^{(j j)}$, and expression (6) for $\Delta_{k, k-1}^{(i j)}$ is definitely proved.

\subsection{Distributed fusion filter}

Once the local least-squares linear filters (Theorem 3.1) and their cross-covariance matrices (Theorem 3.2) have been calculated, the optimal distributed fusion filter is designed as the matrix-weighted linear combination of such local filters that minimizes the mean squared error, according to the following theorem [1].

Theorem 3.3. For $i=1, \ldots, m$, from the local filters, $\widehat{x}_{k / k}^{(i)}$, given in Theorem 3.1 and the cross-covariance matrices, $K_{k / k}^{(i j)}$, given in Theorem 3.2, the distributed fusion filter, weighted by matrices in the linear minimum variance sense, for system (1)-(2) is given by

$$
\widehat{x}_{k / k}^{(D)}=\Xi_{k / k} K_{k / k}^{-1} \widehat{X}_{k / k}, \quad k \geq 1,
$$

where $\widehat{X}_{k / k}=\left(\widehat{x}_{k / k}^{(1) T}, \ldots, \widehat{x}_{k / k}^{(m) T}\right)^{T}, \quad K_{k / k}=\left(K_{k / k}^{(i j)}\right)_{i, j=1, \ldots, m}$ and $\Xi_{k / k}=$ $\left(K_{k / k}^{(11)}, \ldots, K_{k / k}^{(m m)}\right)$.

The error covariance matrices of the distributed fusion filters are computed by

$$
\Sigma_{k / k}^{(D)}=\mathcal{D}_{k}-\Xi_{k / k} K_{k / k}^{-1} \Xi_{k / k}^{T}, \quad k \geq 1
$$

Proof. This proof can be seen in [1], so the details are omitted here. 
Remark: The derivation of the proposed algorithms involves some difficulties associated with the correlation hypothesis of the random parameter matrices (Hypothesis 2), namely:

- The derivation of expression (3) for the matrices $\Phi_{k}^{(i)} \equiv E\left[\left(\widetilde{F}_{k} x_{k}+w_{k}\right) \mu_{k}^{(i) T}\right]$, for which some properties of the conditional expectation must be used to obtain $E\left[\widetilde{F}_{k} \mathcal{D}_{k} H_{k}^{(i) T}\right]$ and $E\left[\widetilde{F}_{k} \mathcal{E}_{k}^{(i)} B_{k}^{(i) T}\right]$ (which are equal to zero when the transition and observation matrices are independent).

- Analogous comments can be made concerning the derivation of $\Psi_{k, k-1}^{(i)} \equiv$ $E\left[\left(\widetilde{H}_{k}^{(i)} x_{k}+B_{k}^{(i)} v_{k}^{(i)}\right) \mu_{k-1}^{(i) T}\right]$, for which we must calculate $E\left[\widetilde{H}_{k}^{(i)} F_{k-1} \mathcal{D}_{k-1} H_{k-1}^{(i) T}\right]$ and $E\left[\widetilde{H}_{k}^{(i)} F_{k-1} \mathcal{E}_{k-1}^{(i)} B_{k-1}^{(i) T}\right]$ (expectations that are straightforward when the transition matrices are independent of the observation matrices).

- The derivation of the cross-covariance matrices $K_{k / k}^{(i j)}$ (Theorem 3.2) also entails some difficulties due to the correlation of the transition matrices. They are mainly associated with obtaining the expectation $\Delta_{k, k-1}^{(i j)} \equiv E\left[H_{k}^{(i)} x_{k} \mu_{k-1}^{(j) T}\right]$ and the calculations involving this expectation.

\section{Numerical example: multi-sensor systems with random delays}

In this section a numerical simulation example is presented. The hypotheses considered in the current paper allow us to apply the results to multi-sensor systems with random failures in data transmission; the purpose of this example is to illustrate our study by considering a two-sensor system with correlated randomly delayed measurements as a particular case.

Let $\left\{x_{k}\right\}_{k \geq 0}$ be a scalar state process which must be estimated from measurements coming from two different sensors, subject to random one-step delays correlated at consecutive sampling times, namely:

$$
\begin{aligned}
& x_{k}=F_{k-1} x_{k-1}+w_{k-1}, \quad k \geq 1, \\
& z_{k}^{(i)}=C^{(i)} x_{k}+v_{k}^{(i)}, \quad k \geq 1, \quad i=1,2, \\
& y_{k}^{(i)}=\left(1-\gamma_{k}^{(i)}\right) z_{k}^{(i)}+\gamma_{k}^{(i)} z_{k-1}^{(i)}, \quad k \geq 2, \quad y_{1}^{(i)}=z_{1}^{(i)}, \quad i=1,2
\end{aligned}
$$

where $F_{k}=0.01\left(\alpha_{k}^{(1)}+\alpha_{k}^{(2)}\right)+0.95$ with $\left\{\alpha_{k}^{(i)}\right\}_{k \geq 1}, i=1,2$, two independent sequences of independent and identically distributed Bernoulli random variables such that $P\left[\alpha_{k}^{(i)}=1\right]=\bar{\alpha}^{(i)}$. Also, $C^{(1)}=0.8$ and $C^{(2)}=0.5$, the additive noises are defined as $w_{k}=0.6\left(\eta_{k}+\eta_{k+1}\right)$ and $v_{k}^{(i)}=c^{(i)} \eta_{k}, i=1,2$, with $c^{(1)}=1, c^{(2)}=0.8$ and $\left\{\eta_{k}\right\}_{k \geq 0}$ is a zero-mean Gaussian white process with variance 0.5.

For $i=1,2$, the phenomenon of delayed measurements is described by the random variables $\gamma_{k}^{(i)}$ which are defined from $\left\{\alpha_{k}^{(i)}\right\}_{k \geq 1}$ as follows:

$$
\gamma_{k}^{(i)}=\alpha_{k+1}^{(i)}\left(1-\alpha_{k}^{(i)}\right), \quad i=1,2
$$

Hence, the variables $\gamma_{k}^{(i)}$ and $\gamma_{s}^{(i)}$ are one-step correlated. 
Then, using the notation

$$
\begin{aligned}
& X_{k} \equiv\left(\begin{array}{c}
x_{k} \\
x_{k-1}
\end{array}\right), \quad k \geq 1, \quad X_{0} \equiv\left(\begin{array}{c}
x_{0} \\
0
\end{array}\right), \quad \mathcal{F}_{k} \equiv\left(\begin{array}{cc}
F_{k} & 0 \\
1 & 0
\end{array}\right), \quad W_{k} \equiv\left(\begin{array}{c}
w_{k} \\
0
\end{array}\right), \quad k \geq 0, \\
& H_{k}^{(i)} \equiv\left\{\begin{array} { c l } 
{ ( C ^ { ( i ) } , 0 ) , } & { k = 1 , } \\
{ ( ( 1 - \gamma _ { k } ^ { ( i ) } ) C ^ { ( i ) } , \gamma _ { k } ^ { ( i ) } C ^ { ( i ) } ) , } & { k \geq 2 , }
\end{array} \quad B _ { k } ^ { ( i ) } \equiv \left\{\begin{array}{cc}
(1,0), & k=1, \\
\left(1-\gamma_{k}^{(i)}, \gamma_{k}^{(i)}\right), & k \geq 2,
\end{array}\right.\right. \\
& V_{k}^{(i)} \equiv\left\{\begin{array}{cc}
\left(v_{1}^{(i)}, 0\right)^{T}, & k=1, \\
\left(v_{k}^{(i)}, v_{k-1}^{(i)}\right)^{T}, & k \geq 2,
\end{array}\right.
\end{aligned}
$$

we can easily observe that the random transition parameter matrices $\left\{F_{k}\right\}_{k \geq 0}$ and measurement matrices $\left\{H_{k}^{(i)}\right\}_{k \geq 1}$ and $\left\{B_{k}^{(i)}\right\}_{k \geq 1}$, as well as the process noises $\left\{W_{k}\right\}_{k \geq 0}$ and $\left\{V_{k}^{(i)}\right\}_{k \geq 1}$, satisfy the correlation hypotheses specified in Section 2.

Thereby, system (7) is rewritten following the structure given by equations (1)-(2):

$$
\begin{aligned}
& X_{k+1}=\mathcal{F}_{k} X_{k}+W_{k}, \quad k \geq 0, \\
& y_{k}^{(i)}=H_{k}^{(i)} X_{k}+B_{k}^{(i)} V_{k}^{(i)}, \quad k \geq 1, \quad i=1,2,
\end{aligned}
$$

and the distributed fusion filtering algorithm proposed can be applied to this system.

To illustrate the accuracy of the proposed algorithm, it was implemented in MATLAB and fifty iterations were run, considering different values of the probabilities $\bar{\alpha}^{(i)}, i=1,2$, for which similar conclusions are derived. Then, the performance of the distributed fusion filtering estimator is illustrated graphically for $\bar{\alpha}^{(1)}=0.5$ and $\bar{\alpha}^{(2)}=0.2$ which yield to the delay probabilities $\bar{\gamma}^{(1)}=0.25$ and $\bar{\gamma}^{(2)}=0.16$.

Firstly, Figure 1 shows the state estimates obtained from the corresponding simulated observations, the local estimates and the proposed distributed filtering estimates. From this figure, it is deduced that all the estimates follow a satisfactory and efficient tracking performance.

Next, the error variances of the local filters and the distributed fusion filter are displayed in Figure 2. From this figure, we can see that the distributed filter has better accuracy than any local one. The local filter obtained from the second sensor is more accurate than that obtained from the first one since the second sensor delay probability is smaller.

\section{Conclusions}

The distributed fusion filtering problem for multi-sensor stochastic systems with correlated random parameter matrices and additive noises in the state and measurement equations has been addressed. Specifically, local least-squares linear filters have been designed from the measurements coming from each single sensor and the crosscovariance matrices between any two local filters have been specified. Then, the distributed fusion filter has been obtained as a matrix-weighted linear combination of the local ones, under the mean squared error criterion. It is worth highlighting that the correlation hypothesis between the transition parameter matrices and the measurements coming from each sensor is useful to consider several general multi-sensor 


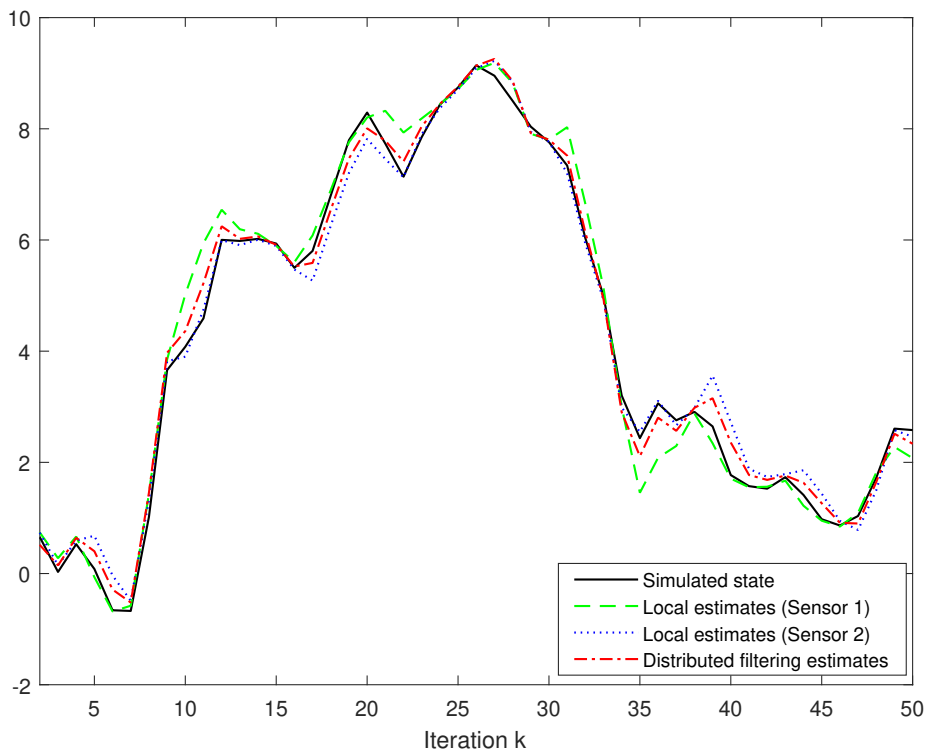

Figure 1. Simulated state, local estimates and proposed distributed filtering estimates.

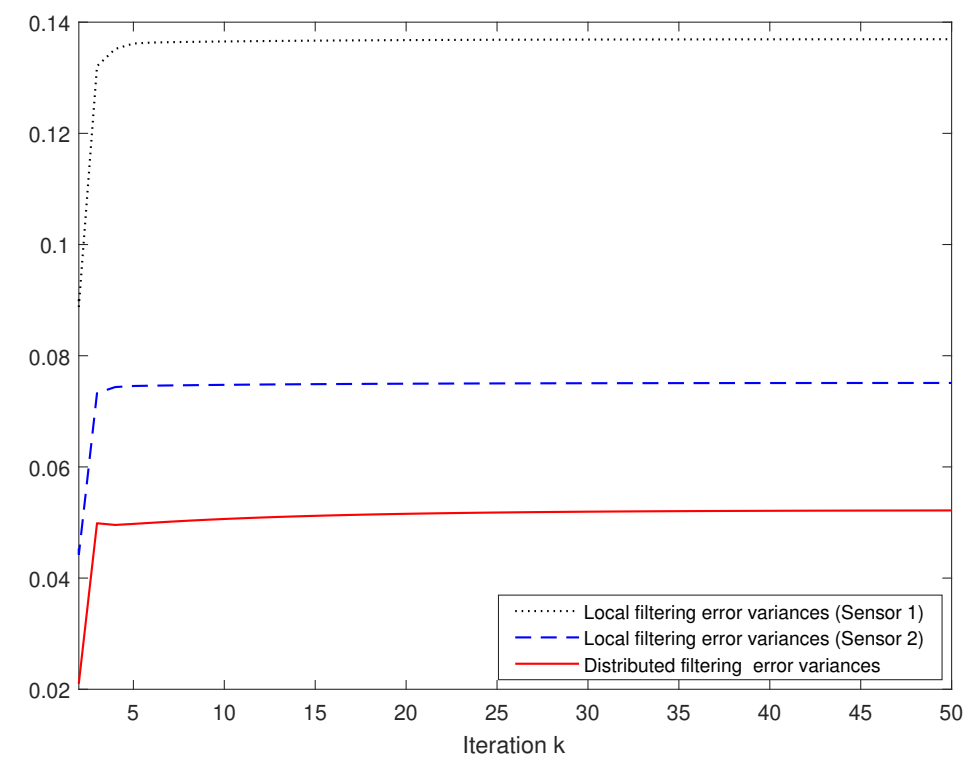

Figure 2. Local and distributed fusion filtering error variances.

systems with different kind of random failures. For example, as we can see in Section 4 , the theoretical results obtained are applicable to multi-sensor systems with random delays. 


\section{Disclosure statement}

No potential conflict of interest was reported by the authors.

\section{Funding}

This work is supported by Ministerio de Economía, Industria y Competitividad, Agencia Estatal de Investigación and Fondo Europeo de Desarrollo Regional (FEDER) (grant no. MTM2017-84199-P).

\section{ORCID}

R. Caballero-Águila http://orcid.org/0000-0001-7659-7649

I. García-Garrido http://orcid.org/0000-0003-3101-4088

J. Linares-Pérez http://orcid.org/0000-0002-6853-555X

\section{References}

[1] R. Caballero-Águila, I. García-Garrido, and J. Linares-Pérez, Distributed fusion filtering in networked systems with random measurement matrices and correlated noises, Discret. Dyn. Nat. Soc. 2015 (2015), Article ID 398605, 10 pages.

[2] R. Caballero-Águila I. García-Garrido, and J. Linares-Pérez, Distributed fusion estimation from measurements with correlated random parameter matrices and noise correlation, Int. J. Comput. Math. (2018), DOI: 10.1080/00207160.2018.1437264.

[3] R. Caballero-Águila, A. Hermoso-Carazo, and J. Linares-Pérez, Fusion estimation using measured outputs with stochastic uncertainties and correlated random transmission delays, Sensors 16 (2016), pp. 1-18.

[4] R. Caballero-Águila, A. Hermoso-Carazo, and J. Linares-Pérez, Fusion estimation using measured outputs with random parameter matrices subject to random delays and packet dropouts, Signal Process. 127 (2016), pp. 12-23.

[5] B. Chen, and W. Zhang, Distributed fusion estimation with missing measurements, random transmission delays and packet dropouts, IEEE Transact. Autom. Control 59 (2014), pp. 1961-1967.

[6] J. Hu, Z. Wang and H. Gao, Recursive filtering with random parameter matrices, multiple fading measurements and correlated noises, Automatica 49 (2013), pp. 3440-3448.

[7] J. Hu, Z. Wang, B. Shen and H. Gao, Quantised recursive filtering for a class of nonlinear systems with multiplicative noises and missing measurements, Int. J. Control 86 (2013), pp. 650-663.

[8] J. Hu, Z. Wang, S. Liu and H. Gao, A variance-constrained approach to recursive state estimation for time-varying complex networks with missing measurements, Automatica, 64 (2016), pp. $155-162$.

[9] W. Li, Y. Jia, and J. Du, Distributed filtering for discrete-time linear systems with fading measurements and time-correlated noise, Digit. Signal Process. 60 (2017), pp. 211-219

[10] J. Linares-Pérez, R. Caballero-Águila, and I. García-Garrido, Optimal linear filter design for systems with correlation in the measurement matrices and noises: recursive algorithm and applications, Int. J. Syst. Sci. 45 (2014), pp. 1548-1562.

[11] L. Liu, A. Yang, X. Tu, M. Fei, and W. Naeem, Distributed weighted fusion estimation for uncertain networked systems with transmission time-delay and cross-correlated noises, Neurocomputing 270 (2017), pp. 54-65. 
[12] F. Peng, and S. Sun, Distributed fusion estimation for multisensor multirate systems with stochastic observation multiplicative noises, Math. Probl. Eng. 2014 (2014), Article ID 373270, 8 pages.

[13] B. Shen, Z. Wang, and Y. Hung, Distributed $H_{\infty}$-consensus filtering in sensor networks with multiple missing measurements: The finite-horizon case, Automatica 46 (2010), pp. $1682-1688$.

[14] S. Sun, H. Lin, J. Ma, and X. Li, Multi-sensor distributed fusion estimation with applications in networked systems: A review paper, Inf. Fusion 38 (2017), pp. 122-134.

[15] S. Sun, F. Peng, J. Ma, and H. Lin, Distributed asynchronous fusion estimator for stochastic uncertain systems with multiple sensors of different fading measurement rates, IEEE Trans. Signal Process. 66 (2018), pp. 641-653.

[16] S. Sun, T. Tian, and L. Honglei, State estimators for systems with random parameter matrices, stochastic nonlinearities, fading measurements and correlated noises, Inf. Sci. 397-398 (2017), pp. 118-136.

[17] T. Tian, S. Sun, and N. Li, Multi-sensor information fusion estimators for stochastic uncertain systems with correlated noises, Inf. Fusion 27 (2016), pp. 126-137.

[18] S. Wang, H. Fang, and X. Liu, Distributed state estimation for stochastic non-linear systems with random delays and packet dropouts, IET Control Theory Appl. 9 (2015), pp. 2657-2665. 